\title{
An Integrated Solution of the Unified Communication System
}

\author{
Balu N. Ilag \\ UC \& Collaboration Expert \\ Juniper Networks, Sunnyvale CA
}

\author{
Ashok Hebbar \\ UC Consultant \\ Bangalore, India
}

\begin{abstract}
The introduction of the enterprise-wide Internet Protocol (IP) infrastructure has made it possible for a variety of services to be hosted on the IP network. An example of this service is the Unified Communications System (UCS). This service enables voice, video, and data to be transmitted over the internet standard on a single network. The improvement of virtualized computer systems allows ease of management of the various components linked to the Unified Communication System. For this reason, there is increased flexibility of the UCS components. The various parts at the data center have greatly helped in achieving a consistent, seamless, secure and highlyavailable telephony service over the enterprise network. In summary, the solution functions as follows; a part of the system and method relies on a data communication network forming a middle part of the distribution network. Telephone communication is usually used either for the earlier or later parts. The system for recovery can acknowledge Voicemail, E-mail, facsimiles and other message types by the one who has subscribed. Cisco Unified Communication system together with Microsoft's solution provides an integrated solution that has improved the efficiency of the business processes.
\end{abstract}

\section{General Terms}

Unified Communication and collaboration, Voice Over IP, Voice Communication.

\section{Keywords}

UCS, Unified Communication System, UC application, IP

\section{INTRODUCTION}

In the past years, the IT industry has witnessed many collaborative software solutions and related tools whose aim is to provide a platform for businesses to expand. In this dynamic fast-moving world, and with the current technological advancement, it is very crucial that companies can access communication tools from a range of communication devices. This kind of access allows people in an organization to be connected no matter where they are. Unified communication technology is one of the leading solutions in the field of IT that has enhanced the efficiency of business processes and people engagement.

\section{UNIFIED COMMUNICATION SYSTEM}

Unified Communication System is a technology that enables the collaboration of elements. (Mantripragada et al. 2012) state that Unified Communication technology provides realtime communication among users including voice, video, messaging, meetings. For example, users can access groupware calendars. With this, they can accept or reject meeting requests through the interface provided. Also, UC users can access as well set up appointments or meetings and also check what is on their schedule. UC Systems even come with a call feature that enables its user's access to real-time control. (Mantripragada et al. 2012). This is possible through call completion features such as automatic call return capabilities, missed call log with call return capabilities, and outbound dialing from the personal address books.

The call feature makes it possible for one to answer the calls or send messages from whatever location they could be. While out of office, an employee can trace the calls made to them, while away (Bradley \& Shah. 2009). Another significant feature provided by this technology is the messaging. As explained by (Mantripragada et al. 2012) the messaging feature delivers user access to voice, and email messages, through a simple interface. This typically occurs via a single interface through the telephone, desktop PC, or mobile device.

The latest generation of the UC systems includes "personal assistants" with speech recognition technology. These assistants as elaborated by (Bradley \& Shah. 2009) help mobile employees manage their communication, including messaging, call control, groupware, and other enterprise data. Therefore, UC technology is the integration of real-time communication services which include instant messaging, online visibility, telephony, audio and video conferencing, integrated voice mail, email, SMS and fax. The UC technology is merely an integration of a wide range of products that offer a consistently unified user-experience across multiple devices and media (Bradley \& Shah. 2009). It also allows for the incorporation through software to business processes. Unified Communication usage increase drastically in every industry, users are moving from phone calls to textbased conversations. Contact Center is one of the industries using VoIP significantly because it provides cost-effective tools to proactively manage the customer engagement process, improve customer service and reduce staff costs. VoIP based contact center solutions include call center software that connects and manages customer interactions across multiple media types, originated from almost any device.

Unified communication technology is very secure and fast. It enables the transmission of data at a very high speed. Each network is at high risk of being attacked by threats (Mantripragada et al. 2012). The vulnerability poses a higher danger to every data that is transmitted on a network. (Bradley $\&$ Shah. 2009) explain that to prevent the risks, information is encrypted. UC connection ensures that the data transmitted through it is encoded. The encryption is very key towards ensuring that data gets safely to the destination. As a result of data encryption, the message is kept private within the network.l

Below are some of benefits given by Unified Communication system

1. UC provides integrated solution including Voice, Video, Content, messaging etc.

2. Cost effective solution when switching from legacy to VoIP based infrastructure 
3. More effective communication in innovative and mobile work contexts.

4. Better audio and video quality when UC solution deploy with help of quality of service (QoS).

\section{UNIFIED COMMINICATION AND ITS APPLICATION}

In today Unified communication market, there are many Unified Communication applications which provides realtime communication and collaboration services which including Instant Messaging, voice and video call, inbound and outbound call routing, jInteractive Voice Recognition, online visibility, voice mail and many more. For this paper have elaborated top industry applications which provides UC solution for on-premise as well as cloud.

\subsection{Cisco Unified Communications}

One of the current UCS technologies is the Cisco Unified Communications. This system incorporates and integrates some of the essential communications technologies. One of them is IP Telephony. Through this technology, voice communications are transmitted over a network using IP standards (Mantripragada et al. 2012). Cisco Unified Communications include a wide array of hardware and software products such as call processing agents, IP phones, voice messaging systems, video devices, and many unique applications. Video telephony is the other technology provided by Cisco Unified Communications. This feature allows real-time video communications and collaboration using the same IP network and calls processing agent (Bradley \& Shah, 2009). Video telephony has made it easy to make video calls. Finally, Cisco Telepresence delivers realtime, face-to-face interactions between people and places in their work and personal lives using advanced visual, audio, and collaboration technologies. Cisco's UC solution is available based on device license and hardware.

\subsection{Microsoft UC and Collaboration Solution}

According to (Pahlavan et al. 2011), Skype for Business and Microsoft's Office 365 is a cloud-based integrated UC solution that connects the team via online hosted voice and video. The reason for this is to establish increased productivity. Microsoft UC and Collaboration solution through Office 365 provides an ever-expanding category of applications which include document sharing, chat-based teamwork, secure email and many more (Pahlavan et al., 2011). Microsoft's Skype for Business has brought audio calling and conferencing capabilities into the cloud.

Research shows that cloud Private Branch Exchange (PBX) which is Microsoft's cloud-based solution allows the control of call features like transfer, hold, voice mail and many others (Pahlavan et al., 2011). Instant messaging and presence are also possible with Microsoft's Skype for Business client. With this solution, the Active Directory and Azure both are integrated to search for resources within an organization. Video conferencing is also possible with this solution. Skype for business as well provides the possibility of an online collaborative meeting. The study by (Bradley \& Shah, 2009) shows that this feature provides the ability to conduct a meeting of up to 250 people. According to (Ilag, B. N, 2018). Microsoft's New Chat-Based Workspace in Office 365, known as Microsoft Teams is successor of Skype for Business which takes, Unified Communication and Collaboration capabilities to next level with providing application sharing, persistent chat, presence, voice and video call in cloud with real-time call qualities dashboard which help to optimize enduser experience and organization to achieve more (Ilag, B. N, 2018). The conferencing functionality is collaborated into the Microsoft. Users can join the sessions by clicking on "Join meeting" button on their calendar reminders which launches the course seamlessly. Microsoft UC solution available as onpremise server license as well as subscription based.

\subsection{Asterisk UC Solution}

Asterisk is an open source PBX platform that is widely used by organization. In fact, many providers of cloud-based PBX solutions use Asterisk to power their service. Asterisk UC solution provides many features that continues to grow year by year. According to (Desmond, P. 2015) Asterisk is an open source system, organization can turn any computer system into a complete communications server. It provides variety of PBX features, including voicemail, IVR, rule-based call routing, automatic call distribution and conference calling. The Asterisk PBX platform is completely free and open source, so organization can deploy it and modify it as they need without having to pay any licensing fees.I

\section{EVOLUTION OF VOICE OVER IP}

With the advancement of technology, most enterprises seek out for applications that can run on converged networks of voice IP and data networks. Unified Communication is one such technology which allows this convergence. This technology allows for the broadcast of data packets over the Internet, from one IP address to another (Pahlavan et al., 2011). In a nutshell, Voice over IP provides the users with the opportunity to not only make calls but also perform other communication activities like call forwarding and voicemail over the Internet. Voice is transformed into digital data. These packets are channeled online through cable lines, IP address or fiber optic.

\subsection{The integration of UC and Voice over IP}

The combination of these two technologies, UC and IP, offers a lot of benefits. Taking a closer look at the functionality and features, it is evident that the new generation of IP-enabled UC systems enable the integration of presence awareness and instant messaging via a standard user interface. Through the cooperation of these various technologies, UC systems are able to route intelligently regarding the information about the user's calendar, personal rules and presence status (Pahlavan et al., 2011). The UC system is then able to direct instant messages to users, alerting them of any other communication or signals of interest.

The design benefits of IP Based UC system are that it is much simpler to integrate, scale and expand (Pahlavan et al., 2011). It is also less expansive to manage. As VoIP is very standardsdriven, the integration of converged applications such as UC is more straightforward and cost-effective given the removal of interface software and hardware components. During the implementation of new IP PBX's, it is straightforward to add Unified communication. According to (Bradley \& Shah, 2009) the addition does not also require some of the integration efforts that were needed to integrate UC on Timedivision multiplexing (TDM) platforms. The whole process is time-saving since it is significantly reduced in a standardsbased, single-protocol environment.

The evolution of VoIP is gradual. Early in the days, there were some concerns which hindered its full adoption. Such matters included poor call quality. This resulted from dial-up 
phone connections that could not handle the data traffic. As explored by (Pahlavan, et al. 2011), a more significant problem was that these lines were part of the public switched telephone network. This meant nobody could use the phone and Internet at the same time. To try and resolve the problem, users added a second landline. It is during this time that companies also began to produce equipment which was able to switch between the internet and telephone. Consumers were also able to replace dial-up connections with broadband. VoIP became more flexible and reliable than landlines and as a result its services became cheaper. Hence, the users were able to contact anyone in the world for next to nothing. UC technology supports contact center workload as well for inbound call center solution helps companies with incoming calls, such as with customer service and tech support. Outbound call center solution can help businesses with market research, lead generation and customer outreach. Telemarketers use outbound call centers. Unified call center solutions can provide both inbound and outbound services for manufacturing companies.

\section{THE FUTURE OF VOICE IP}

Since its adoption, VoIP is rapidly evolving, and its future cannot be predicted. Today, VoIP has become globally significant due to its high use activities by consumers and enterprises. Tomorrow's VoIP is crucial to accommodate many of the users for mobile technology, on-demand calling, and voice among apps (Pahlavan et al. 2011). The migration towards mobile technology is very rampant, and henceforth each new technology should strive towards this. Mobile opportunities such as Google AdWords are the features that consumers are looking out for. They continue to demand responsive customer services in digital experiences for every industry. Lastly, the popularity of voice among Apps infiltrate the market and consumers react, VoIP and real-time web communication are ever popular for remote video conferencing, file transfer, chat, and desktop sharing, to name a few.

\section{CONCLUSION}

As a final analysis of the discussion, Unified Communications technology is very crucial towards the achievement of efficient business process. Asterisk, Cisco's Unified communications technologies as well as Microsoft's UC and Collaboration are among the most widely used components for efficient collaboration, these providers are pushing their products onto the market. The relative innovation of the systems as well as a range of unanswered technical and organizational questions obstruct the spread of UC systems. Therefore, the opportunity exists for new researchers to assist corporations in implementing UC systems using designoriented research studies. In overall the technology allows for faster and reliable communication. It is also very secure, and therefore there is guaranteed safety of each data that is transmitted. With the ever-changing technology, there is need to keep up to date.

\section{ACKNOWLEDGMENTS}

Our thanks to the experts who have contributed towards this paper and researcher who has contributed for UC technology. Special thanks to our family member for their continues support.

\section{REFERENCES}

[1] Mantripragada, Srinivas, Amitava Mukherjee, and Barry Bomzer. (2012) "System and method for unified communications threat management (UCTM) for converged voice, video and multi-media over IP flows." U.S. Patent No. 8,161,540. 17 Apr. 2012.

[2] Bradley, T., \& Shah, S. (2009). Unified communications for dummies. John Wiley \& Sons.

[3] Pahlavan, Kaveh, and Prashant Krishnamurthy (2011). Principles of wireless networks: A unified approach. Prentice Hall PTR, 2011.

[4] Ilag, B N. (2018) "Introducing: Microsoft Teams: Understanding the New Chat-Based Workspace in Office, 2018.

[5] Desmond,P(2015) Blog:http://www.theucbuyer.com/blog /3-options-for-an-open-source-unified-communicationsuc-solution.

[6] Lazar I (2006) Integrating telephony, IM, video and mobility with presence. Business Communications Review (6):28-31. 